\title{
A simple and cost-efficient classification of ballpoint pen inks using digital color analysis
}

Nikolai Yu. Tiuftiakov, Andrey V. Kalinichev, Evgeniia Khairullina, Elizaveta K. Gigiadze, Maria A. Peshkova, Ilya I. Tumkin

Institute of Chemistry, Saint Petersburg State University

7/9 Universitetskaya nab., St. Petersburg 199034, Russia

\section{Supporting Information}

Table S1. Parameters of polynomial fit of Saturation-time ink aging curves of the verification dataset (the values are given for each individual dash).

\begin{tabular}{|c|c|c|c|c|c|}
\hline Ink & Dash \# & $a_{0}$ & $a_{1} \times 10^{4}, h^{-1}$ & $a_{2} \times 10^{9}, h^{-2}$ & $a_{3} \times 10^{15}, h^{-3}$ \\
\hline \multirow{5}{*}{1} & 1 & $-2.98 \pm 0.09$ & $-8.12 \pm 0.07$ & $8.06 \pm 0.14$ & $-29.4 \pm 0.7$ \\
\hline & 2 & $-3.37 \pm 0.12$ & $-8.80 \pm 0.08$ & $8.58 \pm 0.17$ & $-30.3 \pm 0.9$ \\
\hline & 3 & $-3.70 \pm 0.12$ & $-8.88 \pm 0.08$ & $8.83 \pm 0.17$ & $-31.3 \pm 1.0$ \\
\hline & 4 & $-3.17 \pm 0.11$ & $-8.75 \pm 0.08$ & $8.69 \pm 0.17$ & $-31.4 \pm 0.8$ \\
\hline & 5 & $-3.19 \pm 0.11$ & $-8.75 \pm 0.08$ & $8.81 \pm 0.17$ & $-31.5 \pm 0.9$ \\
\hline \multirow{5}{*}{2} & 1 & $0.12 \pm 0.05$ & $-3.78 \pm 0.04$ & $3.39 \pm 0.08$ & $-15.5 \pm 0.4$ \\
\hline & 2 & $0.12 \pm 0.06$ & $-3.78 \pm 0.04$ & $3.31 \pm 0.08$ & $-14.8 \pm 0.4$ \\
\hline & 3 & $0.21 \pm 0.05$ & $-3.77 \pm 0.04$ & $3.40 \pm 0.07$ & $-15.6 \pm 0.4$ \\
\hline & 4 & $0.13 \pm 0.05$ & $-3.81 \pm 0.04$ & $3.42 \pm 0.07$ & $-15.6 \pm 0.4$ \\
\hline & 5 & $0.17 \pm 0.05$ & $-3.77 \pm 0.04$ & $3.30 \pm 0.08$ & $-14.9 \pm 0.4$ \\
\hline \multirow{5}{*}{3} & 1 & $0.75 \pm 0.05$ & $-2.26 \pm 0.04$ & $2.18 \pm 0.07$ & $-9.9 \pm 0.4$ \\
\hline & 2 & $0.88 \pm 0.05$ & $-2.24 \pm 0.04$ & $2.04 \pm 0.07$ & $-8.8 \pm 0.4$ \\
\hline & 3 & $1.04 \pm 0.05$ & $-2.20 \pm 0.04$ & $2.09 \pm 0.07$ & $-9.4 \pm 0.4$ \\
\hline & 4 & $1.00 \pm 0.05$ & $-2.05 \pm 0.04$ & $1.97 \pm 0.07$ & $-9.3 \pm 0.4$ \\
\hline & 5 & $0.85 \pm 0.05$ & $-2.19 \pm 0.04$ & $2.03 \pm 0.07$ & $-9.1 \pm 0.4$ \\
\hline \multirow{5}{*}{4} & 1 & $1.36 \pm 0.06$ & $-2.03 \pm 0.05$ & $2.12 \pm 0.09$ & $-10.3 \pm 0.5$ \\
\hline & 2 & $0.77 \pm 0.06$ & $-2.02 \pm 0.04$ & $2.07 \pm 0.08$ & $-9.9 \pm 0.4$ \\
\hline & 3 & $0.73 \pm 0.06$ & $-1.98 \pm 0.05$ & $2.06 \pm 0.09$ & $-10.0 \pm 0.5$ \\
\hline & 4 & $0.86 \pm 0.05$ & $-1.92 \pm 0.04$ & $1.90 \pm 0.08$ & $-9.0 \pm 0.4$ \\
\hline & 5 & $0.97 \pm 0.05$ & $-1.97 \pm 0.04$ & $2.05 \pm 0.08$ & $-10.0 \pm 0.4$ \\
\hline
\end{tabular}



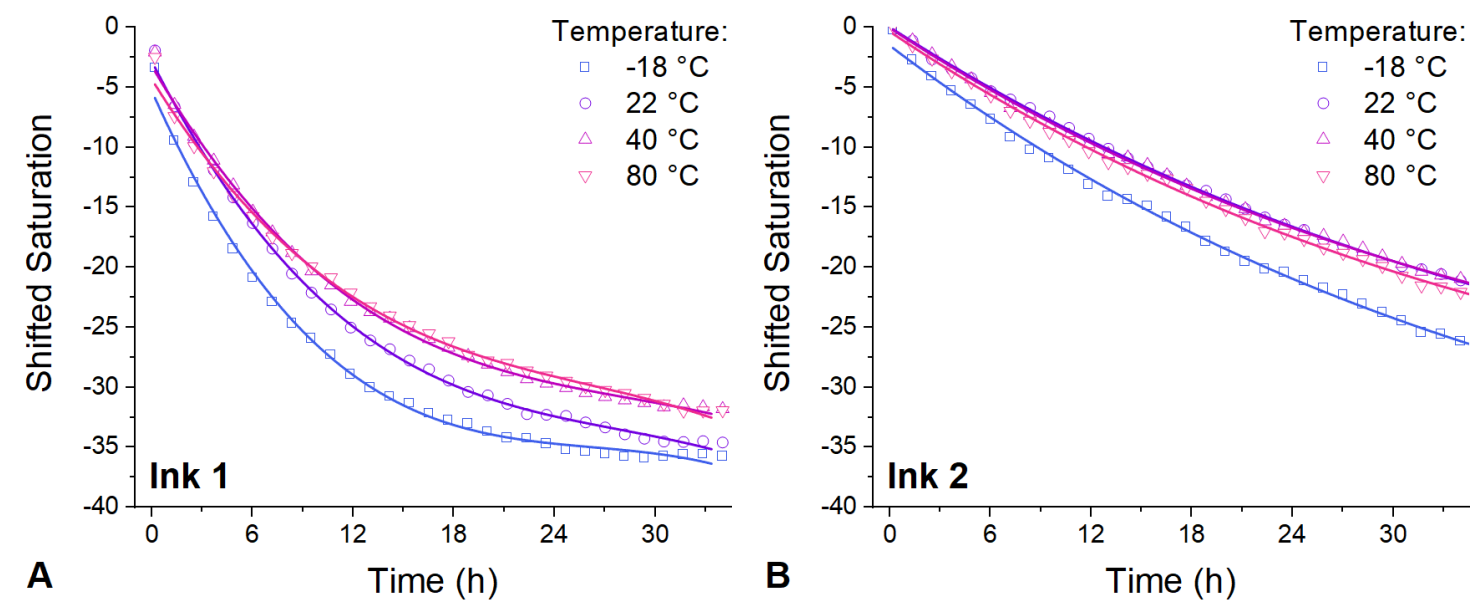

A

Time (h)

B

Time $(\mathrm{h})$
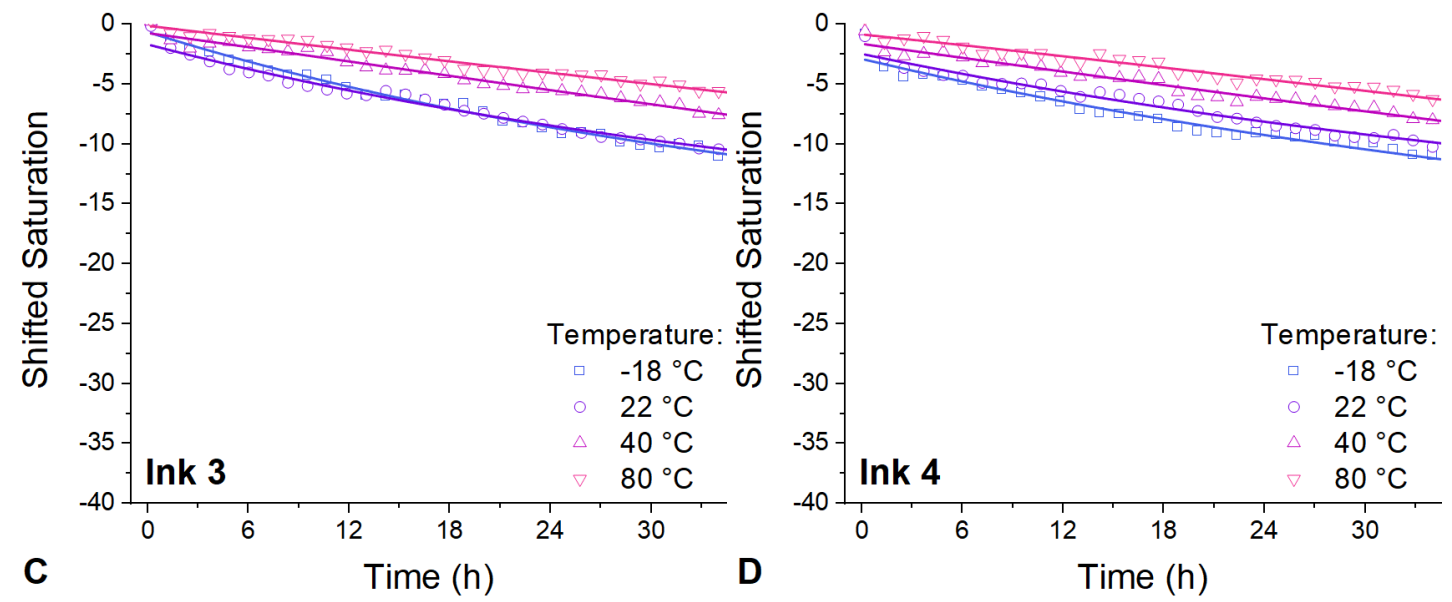

Fig. S1. Polynomial fit of Saturation-time ink aging curves of samples of ink $\mathbf{1}(A), \mathbf{2}(\mathrm{B}), \mathbf{3}$ (C) and 4 (D) stored at four different temperatures $\left(-18^{\circ} \mathrm{C}\right.$, room temperature: $\approx 22^{\circ} \mathrm{C}, 40^{\circ} \mathrm{C}$ and $\left.80^{\circ} \mathrm{C}\right)$ for one week. Symbols - experimental values, solid lines $-3^{\text {rd }}$ degree polynomial fitting function (see Eq. 1). For clarity, only every $6^{\text {th }}$ experimental point is displayed on the graph, the curves are shifted to 0 as the initial value and smoothed (OriginPro 2018, Adjacent-Averaging, Weighted Average, 15 points).

Table S2. Parameters of the polynomial fit of Saturation-time ink aging curves of the ink samples stored at $-18{ }^{\circ} \mathrm{C}$, room temperature: $\approx 22^{\circ} \mathrm{C}, 40^{\circ} \mathrm{C}$ and $80^{\circ} \mathrm{C}$ for one week (the values are given for each individual dash).

\begin{tabular}{|c|c|c|c|c|c|c|}
\hline Temperature, ${ }^{\circ} \mathrm{C}$ & Ink & Dash \# & $a_{0}$ & $a_{1} \times 10^{4}, h^{-1}$ & $a_{2} \times 10^{9}, h^{-2}$ & $a_{3} \times 10^{15}, h^{-3}$ \\
\hline \multirow{10}{*}{-18} & \multirow{5}{*}{1} & 1 & $-4.62 \pm 0.15$ & $-8.82 \pm 0.12$ & $9.11 \pm 0.22$ & $-32.6 \pm 1.2$ \\
\hline & & 2 & $-5.09 \pm 0.16$ & $-9.65 \pm 0.12$ & $10.03 \pm 0.22$ & $-36.1 \pm 1.3$ \\
\hline & & 3 & $-5.24 \pm 0.17$ & $-9.10 \pm 0.12$ & $9.25 \pm 0.25$ & $-32.8 \pm 1.3$ \\
\hline & & 4 & $-4.85 \pm 0.16$ & $-9.08 \pm 0.12$ & $9.39 \pm 0.22$ & $-33.7 \pm 1.2$ \\
\hline & & 5 & $-5.11 \pm 0.15$ & $-8.15 \pm 0.12$ & $8.47 \pm 0.22$ & $-30.5 \pm 1.2$ \\
\hline & \multirow{5}{*}{2} & 1 & $-0.64 \pm 0.07$ & $-3.67 \pm 0.05$ & $2.22 \pm 0.08$ & $-7.9 \pm 0.5$ \\
\hline & & 2 & $-0.39 \pm 0.07$ & $-3.67 \pm 0.05$ & $2.28 \pm 0.11$ & $-8.1 \pm 0.6$ \\
\hline & & 3 & $-0.49 \pm 0.07$ & $-3.72 \pm 0.05$ & $2.44 \pm 0.11$ & $-9.2 \pm 0.6$ \\
\hline & & 4 & $-1.08 \pm 0.08$ & $-3.75 \pm 0.05$ & $2.31 \pm 0.11$ & $-8.3 \pm 0.6$ \\
\hline & & 5 & $-1.21 \pm 0.09$ & $-3.55 \pm 0.07$ & $2.17 \pm 0.14$ & $-7.7 \pm 0.7$ \\
\hline
\end{tabular}




\begin{tabular}{|c|c|c|c|c|c|c|}
\hline & & 1 & $-1.04 \pm 0.10$ & $-1.48 \pm 0.07$ & $0.94 \pm 0.14$ & $-3.6 \pm 0.7$ \\
\hline & & 2 & $-0.46 \pm 0.08$ & $-1.40 \pm 0.07$ & $0.78 \pm 0.11$ & $-2.8 \pm 0.6$ \\
\hline & 3 & 3 & $-0.23 \pm 0.08$ & $-1.15 \pm 0.07$ & $0.31 \pm 0.11$ & $0.0 \pm 0.6$ \\
\hline & & 4 & $-0.14 \pm 0.09$ & $-1.13 \pm 0.07$ & $0.42 \pm 0.11$ & $-1.0 \pm 0.6$ \\
\hline & & 5 & $-1.02 \pm 0.11$ & $-1.47 \pm 0.08$ & $0.78 \pm 0.14$ & $-2.5 \pm 0.8$ \\
\hline & & 1 & $-2.48 \pm 0.11$ & $-1.33 \pm 0.08$ & $0.83 \pm 0.17$ & $-2.5 \pm 0.9$ \\
\hline & & 2 & $-2.40 \pm 0.11$ & $-1.35 \pm 0.08$ & $0.92 \pm 0.14$ & $-3.0 \pm 0.8$ \\
\hline & 4 & 3 & $-2.16 \pm 0.10$ & $-1.30 \pm 0.08$ & $0.78 \pm 0.14$ & $-2.2 \pm 0.8$ \\
\hline & & 4 & $-2.15 \pm 0.11$ & $-1.32 \pm 0.08$ & $0.81 \pm 0.14$ & $-2.2 \pm 0.8$ \\
\hline & & 5 & $-2.38 \pm 0.11$ & $-1.35 \pm 0.08$ & $0.83 \pm 0.17$ & $-2.5 \pm 0.9$ \\
\hline \multirow{20}{*}{22} & \multirow{5}{*}{1} & 1 & $-3.53 \pm 0.11$ & $-8.27 \pm 0.08$ & $7.72 \pm 0.17$ & $-26.7 \pm 0.9$ \\
\hline & & 2 & $-4.43 \pm 0.12$ & $-8.60 \pm 0.08$ & $8.14 \pm 0.17$ & $-28.2 \pm 1.0$ \\
\hline & & 3 & $-4.56 \pm 0.12$ & $-8.35 \pm 0.08$ & $7.81 \pm 0.17$ & $-27.0 \pm 1.0$ \\
\hline & & 4 & $-5.12 \pm 0.14$ & $-8.43 \pm 0.10$ & $8.08 \pm 0.19$ & $-28.4 \pm 1.1$ \\
\hline & & 5 & $-6.29 \pm 0.17$ & $-7.98 \pm 0.12$ & $7.64 \pm 0.22$ & $-26.9 \pm 1.3$ \\
\hline & \multirow{6}{*}{2} & 1 & $-0.15 \pm 0.06$ & $-2.68 \pm 0.04$ & $1.02 \pm 0.08$ & $-2.1 \pm 0.5$ \\
\hline & & 2 & $-0.07 \pm 0.05$ & $-2.41 \pm 0.03$ & $0.67 \pm 0.07$ & $-1.0 \pm 0.4$ \\
\hline & & 3 & $0.09 \pm 0.05$ & $-2.30 \pm 0.04$ & $0.50 \pm 0.07$ & $0.0 \pm 0.4$ \\
\hline & & 4 & $0.01 \pm 0.05$ & $-2.40 \pm 0.04$ & $0.55 \pm 0.07$ & $-0.1 \pm 0.4$ \\
\hline & & 5 & $-0.04 \pm 0.05$ & $-2.58 \pm 0.04$ & $0.84 \pm 0.08$ & $-1.5 \pm 0.4$ \\
\hline & & 1 & $-4.34 \pm 0.13$ & $-1.83 \pm 0.10$ & $1.81 \pm 0.17$ & $-8.3 \pm 1.0$ \\
\hline & \multirow{4}{*}{3} & 2 & $-5.02 \pm 0.15$ & $-1.87 \pm 0.10$ & $1.75 \pm 0.22$ & $-7.6 \pm 1.2$ \\
\hline & & 3 & $-6.18 \pm 0.16$ & $-2.03 \pm 0.12$ & $2.11 \pm 0.22$ & $-9.8 \pm 1.3$ \\
\hline & & 4 & $-6.92 \pm 0.18$ & $-1.97 \pm 0.13$ & $1.97 \pm 0.25$ & $-8.8 \pm 1.4$ \\
\hline & & 5 & $-5.31 \pm 0.15$ & $-2.10 \pm 0.12$ & $2.28 \pm 0.22$ & $-10.6 \pm 1.2$ \\
\hline & \multirow{5}{*}{4} & 1 & $-8.87 \pm 0.23$ & $-1.85 \pm 0.17$ & $2.0 \pm 0.3$ & $-9.7 \pm 1.9$ \\
\hline & & 2 & $-10.31 \pm 0.26$ & $-1.87 \pm 0.18$ & $2.0 \pm 0.4$ & $-9.3 \pm 1.9$ \\
\hline & & 3 & $-10.34 \pm 0.27$ & $-1.98 \pm 0.20$ & $2.2 \pm 0.4$ & $-10.6 \pm 1.9$ \\
\hline & & 4 & $-10.58 \pm 0.27$ & $-2.12 \pm 0.20$ & $2.4 \pm 0.4$ & $-11.6 \pm 1.9$ \\
\hline & & 5 & $-10.28 \pm 0.26$ & $-2.15 \pm 0.20$ & $2.5 \pm 0.4$ & $-12.0 \pm 1.9$ \\
\hline \multirow{11}{*}{40} & \multirow{5}{*}{1} & 1 & $-2.80 \pm 0.10$ & $-6.67 \pm 0.07$ & $5.83 \pm 0.14$ & $-19.0 \pm 0.8$ \\
\hline & & 2 & $-2.78 \pm 0.10$ & $-6.85 \pm 0.08$ & $6.41 \pm 0.15$ & $-22.3 \pm 0.8$ \\
\hline & & 3 & $-3.47 \pm 0.11$ & $-6.72 \pm 0.08$ & $5.71 \pm 0.15$ & $-18.0 \pm 0.8$ \\
\hline & & 4 & $-3.18 \pm 0.10$ & $-7.08 \pm 0.08$ & $6.26 \pm 0.15$ & $-20.5 \pm 0.8$ \\
\hline & & 5 & $-2.99 \pm 0.10$ & $-6.72 \pm 0.07$ & $5.92 \pm 0.14$ & $-19.5 \pm 0.8$ \\
\hline & \multirow{5}{*}{2} & 1 & $0.69 \pm 0.06$ & $-2.96 \pm 0.04$ & $1.79 \pm 0.08$ & $-6.2 \pm 0.4$ \\
\hline & & 2 & $0.24 \pm 0.06$ & $-2.98 \pm 0.05$ & $1.69 \pm 0.09$ & $-5.8 \pm 0.5$ \\
\hline & & 3 & $0.42 \pm 0.06$ & $-3.07 \pm 0.05$ & $1.85 \pm 0.09$ & $-6.6 \pm 0.5$ \\
\hline & & 4 & $0.33 \pm 0.06$ & $-2.99 \pm 0.04$ & $1.63 \pm 0.08$ & $-5.4 \pm 0.5$ \\
\hline & & 5 & $-0.02 \pm 0.06$ & $-2.85 \pm 0.04$ & $1.27 \pm 0.08$ & $-3.4 \pm 0.5$ \\
\hline & 3 & 1 & $-0.57 \pm 0.08$ & $-0.47 \pm 0.06$ & $-0.18 \pm 0.11$ & $1.1 \pm 0.6$ \\
\hline
\end{tabular}




\begin{tabular}{|c|c|c|c|c|c|c|}
\hline & \multirow{9}{*}{4} & 2 & $-0.67 \pm 0.08$ & $-0.38 \pm 0.06$ & $-0.33 \pm 0.12$ & $1.9 \pm 0.6$ \\
\hline & & 3 & $-1.04 \pm 0.10$ & $-0.45 \pm 0.07$ & $-0.27 \pm 0.14$ & $1.7 \pm 0.8$ \\
\hline & & 4 & $-0.99 \pm 0.09$ & $-0.50 \pm 0.06$ & $-0.16 \pm 0.13$ & $1.0 \pm 0.7$ \\
\hline & & 5 & $-0.77 \pm 0.08$ & $-0.58 \pm 0.06$ & $0.03 \pm 0.11$ & $0.0 \pm 0.6$ \\
\hline & & 1 & $-1.62 \pm 0.09$ & $-0.50 \pm 0.07$ & $-0.05 \pm 0.13$ & $0.6 \pm 0.7$ \\
\hline & & 2 & $-1.53 \pm 0.10$ & $-0.50 \pm 0.07$ & $-0.10 \pm 0.14$ & $1.0 \pm 0.7$ \\
\hline & & 3 & $-1.64 \pm 0.10$ & $-0.52 \pm 0.07$ & $-0.09 \pm 0.14$ & $0.8 \pm 0.8$ \\
\hline & & 4 & $-1.73 \pm 0.10$ & $-0.58 \pm 0.07$ & $-0.01 \pm 0.14$ & $0.5 \pm 0.8$ \\
\hline & & 5 & $-1.71 \pm 0.09$ & $-0.46 \pm 0.06$ & $-0.24 \pm 0.13$ & $1.6 \pm 0.7$ \\
\hline \multirow{19}{*}{80} & \multirow{5}{*}{1} & 1 & $-3.95 \pm 0.12$ & $-6.24 \pm 0.09$ & $5.88 \pm 0.17$ & $-21.4 \pm 0.9$ \\
\hline & & 2 & $-4.12 \pm 0.12$ & $-6.56 \pm 0.09$ & $6.12 \pm 0.17$ & $-22.4 \pm 0.9$ \\
\hline & & 3 & $-4.11 \pm 0.12$ & $-6.41 \pm 0.09$ & $5.82 \pm 0.17$ & $-20.5 \pm 0.9$ \\
\hline & & 4 & $-4.01 \pm 0.12$ & $-6.68 \pm 0.09$ & $6.22 \pm 0.17$ & $-22.3 \pm 0.9$ \\
\hline & & 5 & $-4.11 \pm 0.12$ & $-6.55 \pm 0.09$ & $6.16 \pm 0.17$ & $-22.4 \pm 0.9$ \\
\hline & \multirow{5}{*}{2} & 1 & $0.20 \pm 0.07$ & $-2.99 \pm 0.05$ & $1.70 \pm 0.09$ & $-6.2 \pm 0.5$ \\
\hline & & 2 & $0.13 \pm 0.07$ & $-3.25 \pm 0.05$ & $2.31 \pm 0.10$ & $-9.9 \pm 0.5$ \\
\hline & & 3 & $-0.26 \pm 0.07$ & $-2.90 \pm 0.05$ & $1.37 \pm 0.10$ & $-3.9 \pm 0.6$ \\
\hline & & 4 & $-0.23 \pm 0.07$ & $-2.85 \pm 0.05$ & $1.30 \pm 0.10$ & $-3.6 \pm 0.5$ \\
\hline & & 5 & $-0.02 \pm 0.06$ & $-2.93 \pm 0.04$ & $1.44 \pm 0.08$ & $-4.6 \pm 0.5$ \\
\hline & \multirow{5}{*}{3} & 1 & $-0.12 \pm 0.07$ & $-0.30 \pm 0.05$ & $-0.27 \pm 0.10$ & $1.4 \pm 0.6$ \\
\hline & & 2 & $-0.42 \pm 0.08$ & $-0.05 \pm 0.06$ & $-0.84 \pm 0.11$ & $4.8 \pm 0.6$ \\
\hline & & 3 & $-0.55 \pm 0.07$ & $-0.13 \pm 0.05$ & $-0.71 \pm 0.10$ & $4.1 \pm 0.5$ \\
\hline & & 4 & $-0.92 \pm 0.07$ & $-0.47 \pm 0.05$ & $-0.08 \pm 0.10$ & $0.8 \pm 0.6$ \\
\hline & & 5 & $0.08 \pm 0.06$ & $-0.23 \pm 0.04$ & $-0.43 \pm 0.08$ & $2.4 \pm 0.4$ \\
\hline & \multirow{4}{*}{4} & 1 & $-0.49 \pm 0.09$ & $-0.50 \pm 0.07$ & $0.03 \pm 0.13$ & $0.3 \pm 0.7$ \\
\hline & & 2 & $-0.63 \pm 0.09$ & $-0.50 \pm 0.07$ & $0.06 \pm 0.13$ & $-0.1 \pm 0.7$ \\
\hline & & 3 & $-0.95 \pm 0.09$ & $-0.60 \pm 0.06$ & $0.28 \pm 0.12$ & $-1.1 \pm 0.7$ \\
\hline & & 4 & $-0.78 \pm 0.09$ & $-0.54 \pm 0.06$ & $0.09 \pm 0.13$ & $0.0 \pm 0.7$ \\
\hline
\end{tabular}



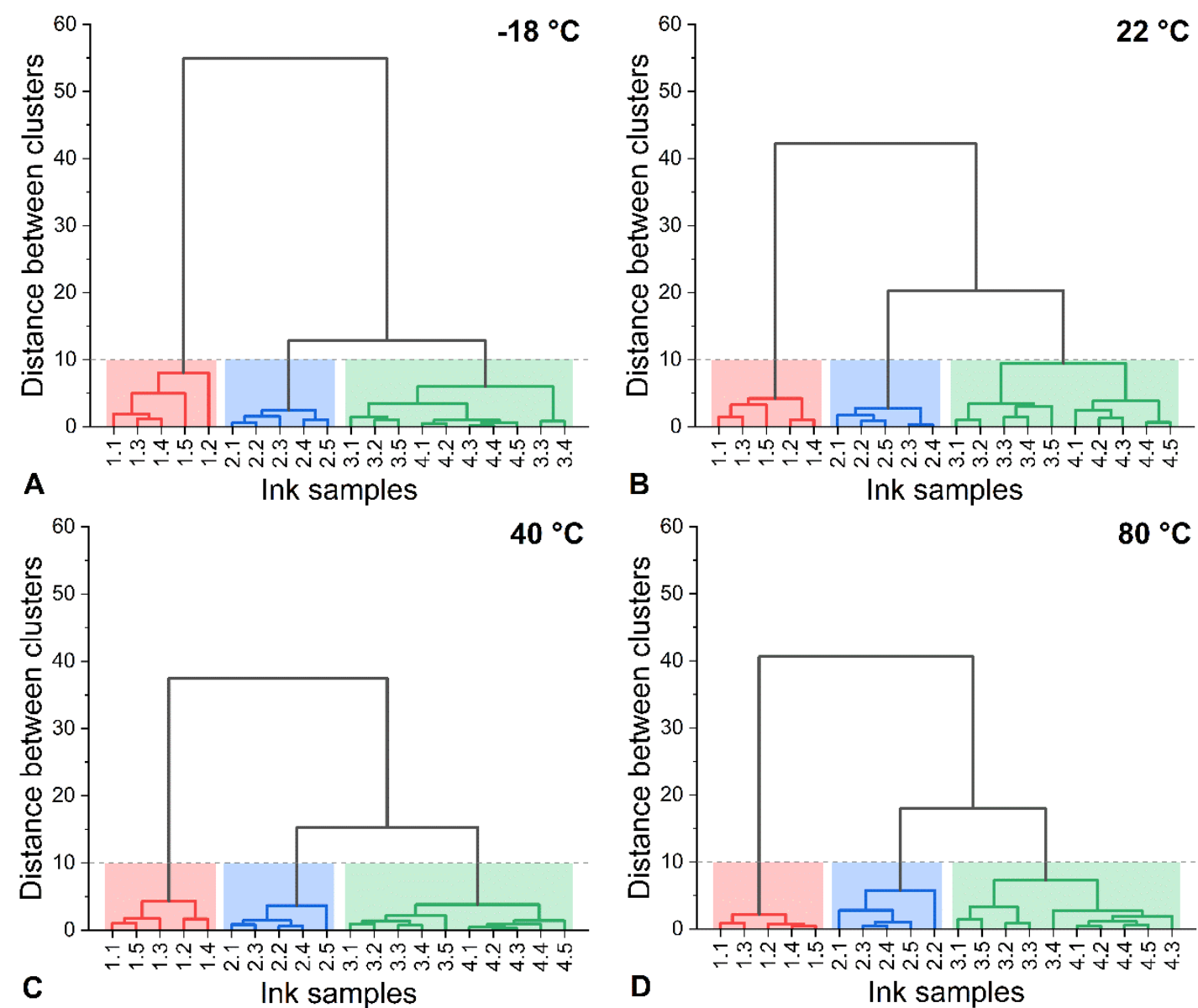

Fig. S2. Dendrograms from the hierarchical cluster analysis based on the polynomial fit (Eq. 1) parameters for the samples stored at $-18{ }^{\circ} \mathrm{C}(\mathrm{A})$, room temperature: $\approx 22{ }^{\circ} \mathrm{C}(\mathrm{B}), 40^{\circ} \mathrm{C}(\mathrm{C})$ and 80 ${ }^{\circ} \mathrm{C}(\mathrm{D})$; the data for each individual dash is shown. 